\title{
Research on the Marketing Strategy of Burgeoning Sugar-Free Beverages-Taking Yuanqi Forest as an Example
}

\author{
Yizhuo Chang ${ }^{1}$ \\ ${ }^{1}$ Zhengzhou Foreign Language School, Zhengzhou, China
}

\begin{abstract}
The appearance of sparkling water has set off a new wave in the beverage industry in China, with the annual sales volume increasing by almost $200 \%$ in 2019 , compared with the 2018 . The market share has risen to fourth place in the beverage market, becoming a new beverage that is only lower than water, carbonated drinks, and tea drinks. YuanQi Forest occupies a large market share in Chinese market, and its products are quite competitive. Therefore, it is of great theoretical and practical significance to study the marketing strategy of the YuanQi Forest at the present stage. This paper mainly adopts SWOT analysis, PEST analysis, and other marketing theories to analyze this kind of situation. The author aims to provide general rules for enterprises engaged in bubble water production and marketing for reference, and provides practical and theoretical guidance for Chinese enterprises to carry out and expand this industry. Through analysis, it can be concluded that YuanQi Forest has three marketing methods, which are product strategy, channel strategy and big data marketing. What is more, Yuanqi Forest has some drawbacks, such as channel construction, lack of supply chain, difficulty in market subsidence, and other problems.
\end{abstract}

\section{INTRODUCTION}

China's bubble water market started in the early 21 st century, with only 20 years of development. Under such circumstances, most of the mainstream bubble water brands in the market come from Europe and America, such as Paris Water. This has led to a single type of bubble water market in China. However, foreign imported products involve a series of factors such as tariff, freight, and labor cost, which tend to be of high price and low market penetration rate, making it challenging to cover the whole domestic market. At the same time, it cannot satisfy the particular taste of some Chinese consumers. As a result, the development of China's bubble water has almost come to a standstill. However, in the past five years, as urban residents' income increases, people started to pursue a healthy and high-quality life [1]. A series of high-end drinks, such as sparkling water, is therefore widespread. In addition to the Paris bubble water on the market, there are also new products such as Xixiao tea, Yuanqi forest. In the current bubble water market, there are still many gaps in market share without a dominant phenomenon. But investors and foreign companies are piling in, and competition is growing fierce. Complexity can radically change the behavior [2]. In such a complex environment, how to use the limited resources to seize the market quota and develop the correct marketing strategy are the critical factors for success. Only by conducting a comprehensive analysis of the market and adopting appropriate analysis tools, and formulating marketing strategies, can YuanQi Forest have a higher chance to seize the market opportunities. Therefore, it is essential to analyze the marketing strategy of Yuanqi Forest.

This paper mainly summarizes SWOT and PEST theories, which lay the theoretical foundation for the marketing environment analysis and marketing strategy proposal in the following chapters of this paper, as well as the supporting part of the follow-up work.

\section{ANALYSIS OF THE MARKETING ENVIRONMENT OF YUANQI FOREST}

\subsection{External Environment of Yuanqi Forest-PEST analysis}

\subsubsection{Political factors}

The political and legal environment has a significant macro-environmental impact on enterprises' marketing strategy [3]. Politics is just like a tangible hand that can change the marketing strategy of a company according to the situation, while the law is the code of conduct for making the strategy. Both of them have a profound impact on the operation and development of enterprises. In the early days of the development of sparkling water, due to tariff barriers and incomplete open policies, sugar-free sparkling water sold at a high price, which made it difficult for sugar-free sparkling water of European origin to enter the sight of ordinary people. As a result, the market penetration rate of bubble water is not high. Simultaneously, as the production and sale of 
counterfeit goods have not been good governed, the public also has doubts about the quality of the bubbling water. In recent years, however, with the acceleration of the Chinese government's reform and development, more foreign investment has been able to enter the Chinese market. Accordingly, the background of the bubble water business has gradually improved and intensified competition between the market.

\subsubsection{Economic factors}

With the rapid development of China's economy, people are more yearning for a high-quality and healthy life. With the average annual growth rate of $6.1 \%$ of GDP per capita, the Engel's coefficient decreases. Therefore, food and clothing problems do not need to be considered. People tend to eat healthier and tastier meals. At the same time, with the popularity of wages and credit cards, people's purchasing power has increased significantly, with year-on-year growth of $8.9 \%$ in 2019 , which is an increase of $92.3 \%$ from 2010. China's development trend has shifted from manufacturing to services. Over the past five years, consumer demand for diet drinks has increased dramatically, and the outlook for the diet drink market is optimistic. According to relevant data, the beverage market from 2010 to 2012 is a golden period for the growth of the beverage market, and the growth of bottled water and carbonated drinks has been sluggish, showing negative growth for two consecutive years. New beverage growth in the past two years is stable in a steady rise.

\subsubsection{Socio-cultural factors}

With the rapid development of society, a variety of beverage flavors and new packaging has appeared a lot. Therefore, it is complicated to design a novel beverage package that is different from previous ones. However, consumers tend to have a higher degree of liking for new packaging. In such situations, consumers are willing to try new packaging types of drinks. Simultaneously, with the development of the economy, people's consumption of psychology has also changed. People tend to pursue higher taste and healthier drinks, with little emphasis on price. There are also some consumer groups through brand linkage and willing to buy. Therefore, the change of consumer psychology stimulates enterprises to make more significant innovation.

\subsubsection{Technological factors}

As countries continue to raise income levels, more and more marketing production and consumption become possible. The quest for natural, healthy, and tasty drinks is on the rise. In recent years, people pay more and more attention to the diversity of beverage variety structure. Consequently, how to reduce artificial food additives and improve production efficiency has become one of the main problems for beverage manufacturers. The beverage industry has developed a sophisticated response to the difficulties of aseptic filling in previous years. From traditional hot filling to cold filling, packaging has also evolved from conventional Bisping and tin cans to PET packaging [4]. Because of the development of production technology, the production process's cost is reduced so that enterprises can spend more experience in product research and development.

\subsection{SWOT Analysis of YuanQi Forest}

\subsubsection{Advantages}

2.2.1.1 As the first local Chinese enterprise operating sugar-free sparkling water in China, YuanQi Forest Beverage entered the market at an early time and possessed a large number of market quotas and loyal consumer groups, occupying a market share of more than $40 \%$. Therefore, consumers have a high reputation and loyalty for Yuanqi Forest beverage.

2.2.1.2 Compared with the xylitol added by the original sparkling water market, the addition of erythritol to YuanQi Forest beverage created a market precedent. Erythritol is healthier than xylitol, but most bubble water manufacturers are reluctant to spend a lot of energy and money on research and development because of the higher extraction process.

2.2.1.3 With a perfect sales channel system, all levels of the market have a good sales network and high-quality dealer groups.

\subsubsection{Disadvantages}

2.2.2.1 Compared with central cities and prefecture-level cities, small towns, counties, suburbs, and other areas have not been well promoted.

2.2.2.2 More and more fizzy drinks companies have sprung up and copied each other, resulting in loss of profits and fierce competition.

\subsubsection{Opportunities}

2.2.3.1 With the improvement of living standards of urban and rural residents, people's consumption habits are gradually changing, and the people gradually favor sugar-free sparkling water drinks [5]. This provides an excellent opportunity for the development of the sugar-free fizzy water beverage industry, especially for YuanQi forest enterprises.

2.2.3.2 From the life cycle theory, unsweetened fizzy water beverages are in the growth stage of products, and there is great potential in the Chinese fizzy water market. Besides, the diversity of consumer tastes and curiosity provides a market space for the development of new products, while China's urban and rural markets have 
considerable growth potential. This is the potential space

for the future development of the Yuanqi Forest.

TABLE I. SWOT MATRIX ANALYSIS OF YUANQI FOREST

\begin{tabular}{|l|l|}
\hline Opportunities & Threats \\
\hline $\begin{array}{l}\text { 1 Economic development } \\
\text { 2 Changes in consumption habits }\end{array}$ Broad market & 2 Internatives \\
& \\
\hline Strengths & Weaknesses \\
\hline $\begin{array}{l}\text { 1 Market advantage } \\
2 \text { Technical advantages } \\
\text { S Stable sales channels } \\
\text { 4 Innovation ability }\end{array}$ & 1 Small coverage \\
\hline
\end{tabular}

\section{MaRketing Strategy of YuANQI Forest}

\subsection{Product Strategy}

\subsubsection{Product concept}

The central concept of the Yuanqi Forest diet drink is "0 sugar, 0 fat and 0 calories". According to the China Business Industry Research Institute, 80 percent of Chinese consumers pay attention to the composition of food and drinks, especially the proportion of sugar in beverages, in the trend of health. In the face of health demands, consumers have to consider reducing their direct intake of sugar. At this time, "0 sugar, 0 fat, and 0 calories" is like telling healthy consumers who want to be thin but also want to be sweet: "Eat me, not fat," to satisfy their food and drink at the same time, alleviate their worries about too much sugar intake [6].

\subsubsection{Channel Strategy—Distribution strategy}

In the marketing plan of YuanQi Forest, the underwriter's main body is usually the major convenience stores. Besides providing a better dining environment, convenience stores can offer products that small stores do not, such as bread with a shorter shelf life, freshly ground coffee, cooked food such as fish-egg cart noodles, and dairy products that need to be transported in the cold chain [7]. Taking 711 as an example, they will have specialized equipment such as milk cabinet, hot cabinet, coffee machine, microwave oven, and cooked food cabinet, and a perfect cold chain logistics and storage systems. The China Chain Management Association released the list of the top 100 Chain stores in China 2018 on May 9. Among the high 100 chain stores, the growth rate of convenience stores is far ahead: the sales scale of the top 100 stores increased $21.1 \%$ year on year, the number of stores increased $18.0 \%$, and 11,494 new stores, accounting for $62.5 \%$ of the total number of new stores of the top 100 stores. In contrast, the sales of the top 100 enterprises, which mainly operate supermarkets, increased by $2.5 \%$ on average, and the number of stores increased by $3.6 \%$ on average, both significantly lower than the average growth rate of the top 100 enterprises.

\subsubsection{Big data marketing}

Data is intelligence, and decision-making requires intelligence. Error correction requires knowledge, and players with more information will undoubtedly have more initiative. The data and knowledge of prediction are the main cause of marketing behavior and firm performance [8]. Because the garage "disruptive innovation" does not see more, most of the time, the industry players are in the same category in the competition. The product differentiation is the so-called "innovation," so who was the first to develop the market, who will be able to grab the consumer's mind, others even experience are not inferior, it is easy to be regarded as "knock-off." Accurately, in the field of consumer goods, YuanQi Forest can understand industry data more frequently and timely, judge "explosive products" and the market first, and grasp the promotion opportunity and distribution strength more accurately. The reason why YuanQi Forest started to build its factory was to achieve a higher response rate than the OEM model. That is to say, the player who can occupy the market realizes the potential of the product to be "explosive" and delivers the product to consumers at the fastest speed, but not necessarily the original developer of the product. This idea has something in common with Tencent's micro innovation concept.

\subsection{Disadvantages of marketing strategy}

\subsubsection{Lack of supply chain}

The asset-light model of industrial production, namely, YuanQi Forest, has no independent supply chain and relies on the OEM production of Jianlibao and Toyang drinks. The company recently completed its first self-built production base in Chuzhou, Anhui province, and will have three high-speed production lines capable of producing tea, sparkling soda, milk tea, and functional drinks with an annual output of 450 million bottles. But this rate of production cannot meet the needs of normal operation or further expansion. This OEM mode is normal in the food and beverage field, and similar operations exist in brands such as Three Squirrels, Gourmet Shop, and Coca-Cola [9]. This mode of operation can reduce the upfront investment and improve the return rate. However, 
it also causes a series of problems such as shortage of supply and uneven quality.

\subsubsection{Small market coverage}

Research has shown that Marketing Turbulence impacted on production development and production success Yuanqi Forest bubble water is targeted at young and fashionable consumers [10]. From the perspective of market promotion, the focus of promotion should be in large and medium-sized cities. According to the survey, the cities with a high market penetration of sugar-free bubble water are mainly in economically developed cities. They are related to the per capita GDP of residents, such as Beijing, Shanghai, Shenzhen, Xiamen, Dalian, and other large and medium-sized cities, with a relatively high proportion. All of them are over $40 \%$. In some small towns and rural areas, like Baoji or Jinhua, the prevalence of YuanQi Forest is lower, which is lower than 25\%. In contrast, a common mineral water brand called Nongfu Spring, a popular water brand, has larger market coverage than that of Yuanqi Forest. Small towns and villages have tremendous market potential, low distribution pressure, low entry cost, accessible communication and management, and a relatively reasonable proportion of input and output [11]. Therefore, whether companies can control the vast rural terminal market determines whether companies can get more income. YuanQi Forest in the second and third markets is powerless and not so influential.

TABLE II. MARKETING BEVERAGE OF TWO DIFFERENT BEVERAGE BRANDS*

\begin{tabular}{|l|l|l|}
\hline $\begin{array}{l}\text { Name of cities } \\
\text { Name of beverage }\end{array}$ & Yuanqi Forest & Nongfu Spring \\
\hline Beijing & $62.3 \%$ & $97.3 \%$ \\
\hline Shanghai & $59.7 \%$ & $981 \%$ \\
\hline Shenzhen & $61.2 \%$ & $93.9 \%$ \\
\hline Guangzhou & $53.1 \%$ & $95.2 \%$ \\
\hline Chendu & $42.5 \%$ & $94.8 \%$ \\
\hline Wuhan & $55.7 \%$ & $95.1 \%$ \\
\hline Jinan & $46.2 \%$ & $91.1 \%$ \\
\hline Zhengzhou & $51.2 \%$ & $96.2 \%$ \\
\hline Dalian & $40.9 \%$ & $89.1 \%$ \\
\hline Hefei & $22.1 \%$ & $90.2 \%$ \\
\hline Xinyang & $25.4 \%$ & $88.9 \%$ \\
\hline Baoji & $19.2 \%$ & $83.3 \%$ \\
\hline Huaihua & $11.2 \%$ & $84.5 \%$ \\
\hline Wuzhou & $9.0 \%$ & $82.4 \%$ \\
\hline Qujing & $5.7 \%$ & $79.3 \%$ \\
\hline Jinhua & $20.2 \%$ & $82.2 \%$ \\
\hline
\end{tabular}

* This survey is conducted in 2019. The margin of error is $5 \%$.

\section{ConClusion}

Marketing has become an essential factor for determining the destiny of Chinese enterprises, and some people even put forward that marketing is the soul and future of enterprises. In this paper, bubble water enterprises' marketing strategy is analyzed, and the author hopes that similar enterprises can draw lessons from and provide theoretical guidance. Due to limitations in access to information and knowledge, analysis in this paper may be not comprehensive and objective, which are the deficiencies of this study.

\section{ACKNOWLEDGMENT}

This thesis was completed under the guidance of Professor Juanjuan Zhang. The professor's profound professional knowledge, serious scientific attitude, and rigorous scholarship have had a profound impact on me. It made me establish ambitious academic goals. I would like to extend my sincerest and heartfelt thanks to Juanjuan Zhang! In addition, the successful completion of this paper is inseparable from the care and help of the teachers. I would also like to thank Teacher Jane and Allison for their guidance and help. They have done a lot of work on this topic and helped me overcome difficulties one by one. Thank them very much! 


\section{REFERENCES}

1. W. X. Zhang, Advanced Marketing [M Lixin Accounting Press, 2010, 35-36

2. West, Wisdom in numbers. ScientificAmerican, 308(5), 2013, 14-14

3. J. Le, Research on unified tea beverage marketing strategy, Southwest Jiaotong University, 2008, 23-24

4. $\mathrm{H} . \mathrm{Mu}$, Research on Beverage Marketing Strategy-Take Jiaduobao as an example, Soochow University, 2014, 32-33

5. L. Juan, Modern Enterprise Brand Development Strategy [M]. Nanjing University Press, 2012

6. H. H. Knight, Innovativeness: its antecedents and impacton business performance. Ind. Mark. Manag, 2004, 429-438

7. X. Zhang. Marketing Management [M]. Shanghai University of Finance and Economics Press, 2008, 78-78

8. Nickerson, Zenger, A knowledge-based theory of the firm: The problem-solving perspective. Organization Science, 2004, 617-618

9. TechAmerica Foundation's Federal Big Data Commission, 2012,21-21

10. Moorman, Christine, Anne S. Miner, The Impact of Organizational Memory on New Product Performance and Creativity, Journal of Marketing Research, 1997, 91-92

11. J. Wen, Current status and future development direction of functional beverage market [J]. Food and Fermentation Industry 2004, 43-44 